\title{
GINECOPATIAS TUMORALES EN LA NIÑEZ
}

Dr. Guillermo Valencia Piedrahíta

El material humano motivo de nuestro estudio lo obtuvimos en el Hospital de San Juan de Dios, Instituto Nacional de Cancerología y en el Hospital de La Misericordia, instituciones todas de esta ciudad; en la revisión de 1.533 exámenes histológicos - con dos casos hallados- de la estadística particular del distinguido patólogo Egon Lichtenberger, un caso de clientela privada del profesor Arturo Aparicio Jaramillo, otro caso de clientela privada del profesor Guillermo López Escobar y, por último, un caso de referencia personal del doctor Eduardo Acosta Bendek, de Barranquilla, y a quienes agradecemos su gentileza por permitir incluirlos en este trabajo.

Consultamos además a todos los miembros del personal científico del Servicio de Ginecología del Hospital de San Juan de Dios, quienes no han tenido casos de esta naturaleza.

Por haber considerado más exacto el examen histo-patológico que el simple diagnóstico clínico, iniciamos la revisión estadística en los respectivos Departamentos de Anatomía Patológica de las instituciones antes nombradas y más tarde la revisión de las historias clínicas correspondientes.

En el Hospital de San Juan de Dios, comenzó a hacerse en forma sistemática el estudio histo-patológico de las piezas quirúrgicas y de autopsias, el 30 de abril de 1933, por el profesor Manuel Sánchez Herrera, quien estuvo hasta febrero de 1953 al frente del Departamento de Anatomía Patológica, realizándose 13.898 exámenes histo-patológicos entre biopsias y material de autopsias. Allí se conservan las placas, bloques de parafina, protocolos, archivos clasificados, etc., de todos estos casos, valioso y unico material de consulta que ha quedado de los años anteriores, ya que en la nueva organización del hospital se desecharon todas las historias clínicas existentes y se empezaron a archivar desde el mes de agosto de 1952, motivo por el cual -y no será culpa ruestra - nos vemos obligados en algunos casos a transcribir sola- 
mente los datos que aparecen en el protocolo de la anatomíapatológica.

Desde el mes de febrero de 1953, dirige la sección de anatomía-patológica del nuevo Hospital de San Juan de Dios, el doctor Egon Lichtenberger, donde se realizaron 1.712 exámenes histopatológicos en 1953, y hasta el momento de escribir este trabajo se habian realizado en este año 1.873 exámenes. En conjunto hay un total de cerca de 18.000 exámenes histo-patológicos, en un lapso de ventiún años, comprendido de 1933 a 1954 .

Encontramos doce casos de tumores del aparato genital femenino, incluyendo el periodo variable de la pubertad.

En el Instituto Nacional de Cancerología se están haciendo estudios histopatológicos desde su fundación en 1934. Hasta el momento se han realizado cerca de 35.000 exámenes, incluyendo la multitud de biopsias que son enviadas de todas partes del país. Allí encontramos quince casos relativos al estudio que nos ocupa. El Departamento de Anatomía Patológica está actualmente a cargo de los doctores M. Mariño Zuleta y A. Méndez Lemaitre.

Por algunas dificultades técnicas, no siempre es fácil aprovechar adecuadamente el valioso y caudaloso material que reposa en los archivos del Instituto.

En el Hospital de La Misericordia, el doctor A. A. Romero Torres está por cuenta de la Facultad de Medicina, encargado de practicar los estudios anatomopatológicos. Sólo desde el año de 1948 se están realizando estos estudios sistemáticamente, pues antes se enviaban las piezas al Instituto de Radium u otros laboratorios o no se hacian dichos exámenes. Hasta la fecha se ha lievado a cabo un total aproximado de 900 exámenes, de los cuales encontramos siete casos de ginecopatias tumorales en las niñas.

En total, hicimos aproximadamente la revisión cuidadosa, hoja por hoja, de cerca de 55.000 protocolos.

Las placas histológicas fueron revisadas para confirmar 0 infirmar el diagnóstico por los patólogos mencionados.

Como se desprende, naturalmente, ha sido muy laborioso para nosotros lograr recopilar 39 casos, que presentamos al estudio en el curso de este trabajo.

En el Hospital de San Juan de Dios se han presentado 1.622 tumores del aparato genital femenino -incluyendo todas las edades- y como encontramos 12 casos en niñas, se deduce una frecuencia del $0.703 \%$, es decir, cerca del $1 \%$. En el Instituto Nacional de Cancerología no fue posible sacar esta frecuencia, de- 
bido al sistema de clasificación empleado, ya que, por ejemplo, si queremos buscar sarcomas del aparato genital, habría que revisar las historias de sarcomas de todo el organismo, lo cual implicaría lógicamente a la larga, una revisión de todas las historias.

En el Hospital de La Misericordia, es obvio, no se pudo hacer tal deducción.

Casi todos los autores coinciden en admitir que los tumores en niños son más frecuentes de lo que generalmente se cree y en lc que hace relación a los tumores del aparato genital femenino, hacemos especial hincapié en este trabajo, sobre su frecuencia. En la minuciosa búsqueda que hemos realizado, encontrar 39 casos, nos ha parecido ciertamente una cifra elevada. Correspondería a una frecuencia del $1 \%$ aproximadamente, en relación a todos los tumores genitales de la mujer, como ya vimos. En cuanto a neoplasias malignas se refiere, por haber encontrado 16 casos, deducimos una frecuencia del $41 \%$, en relación a todos los casos que hallamos. Dargeon (10) comunica 25 casos ginecológicos entre 1.700 casos de cáncer infantil.

Por otra parte en los niños, tumores de alta malignidad histológica, evolucionan benignamente desde el punto de vista clínico y aunque en general son de crecimiento más rápido, con una terapia adecuada se pueden controlar más fácilmente.

No se deben enfocar con el mismo criterio las neoplasias del adulto y las del niño; hay que dar con sus puntos de semejanza y divergencia y tratar de buscar una terapia adecuada para deducir un pronóstico de acuerdo con la patología de cada tumor.

En las estadisticas presentadas por Pack (11), el cáncer es la tercera gran causa de muerte de todos los niños en los Estados Unidos, de uno a catorce años, siendo superada sólo por accidentes y pneumonía y la segunda causa de muerte de cinco a catorce años, siendo sólo superada por accidentes. En los Estados Unidos, mueren de cáncer aproximadamente 2.000 niños por año.

Estos datos nos sirven únicamente para darnos una idea global, puesto que a nuestro trabajo sólo interesan las neoplasias genitales femeninas de la niñez, aun cuando también son de utilidad para llamar la atención sobre lo inadvertidas que pasan para el médico las neoplasias de la niñez y también hacer notar de los pediatras, ginecólogos y aun obstetras, la importancia de pensar siempre en las lesiones tumorales en la consideración del diagnóstico diferencial, ya que si son diagnosticadas oportunamente, podrían, quizá, ser curadas. 
A diferencia del adulto, el carcinoma es raro en los niños, y son más frecuentes los sarcomas, embriomas y tumores mixtos. El por qué de la mayor frecuencia de sarcomas en niños, no está claramente entendido. Ha sido demostrado que la irritación crónica produce ciertos sarcomas como el de las escaras de quemadura; no se sabe si los factores irritativos excitan la formación neoplásica durante la vida prenatal.

En una publicación de Dargeon (Op. cit.), de 1.700 casos de cáncer infantil, encontró 25 ginecológicos, ocupando el noveno lugar en frecuencia, de donde se deduce un porcentaje de uno con cuarenta por ciento $(1.40 \%)$.

Los niños toleran los procedimientos operatorios radicales notoriamente bien, de ahí que ninguno debe ser privado de un intento quirúrgico, curativo. La llamada inoperabilidad por "fragilidad del infante" usualmente refleja fragilidad del cirujano (Pack. Op. cit.), y aconseja el mismo autor que el cirujano puede ser en la mayoría de los casos más conservador, siendo radical desde el principio.

Por otra parte, algunos tumores radio-resistentes según criterio histológico, no lo son clínicamente en los niños, lo mismo que tumores histológicamente benignos — quistes dermoides- se pueden manifestar como malignos.

Es erróneo creer que los sarcomas sólo dan metástasis por vía sanguínea; se debe por lo tanto hacer la extracción de los gánglios que drenan el segmento anatómico en el cual se asienta el tumor; este concepto toma más importancia en la niñez, en donde el elemento embrionario del sarcoma le hace comportarse en forma muy especial.

\section{ONCOLOGIA GENITAL FEMENINA.- EMBRIOLOGIA}

En todo el tracto genital femenino, desde la vulva hasta los ovarios, se pueden presentar diversas clases de neoplasias, pero en general hay algunas que tienen predilección por la temprana edact y que rara vez se presentarian en adultos. En igual forma, pero inversamente, las neoplasias más comunes de la mujer adulta no se presentan o serían afortunados hallazgos en la niñez. Por ejemplo, el carcinoma del cuello o del cuerpo uterino, de tan alta frecuencia en la mujer adulta, es extraordinariamente raro en la niña. Igualmente el sarcoma - ya es sabido- tiene especial predilección por los tejidos jóvenes y el aparato genital de la niña 
no escapa a esta neoplasia. Adelante veremos, que tumores benignos como el angioma congénito de los genitales externos, son de frecuencia considerable en la niña.

Nos llama la atención, en cuanto a la vulva se refiere, que los dos principales tipos de neoplasias, el carcinoma y el sarcoma, tienen preferencia por los dos extremos de la vida, el carcinoma en las ancianas y el sarcoma en las niñas. Queremos hacer resaltar igualmente, que la mayor frecuencia de lesiones tumorales en las niñas, está en los dos extremos del tracto genital: los genitales extremos y los ovarios. La vagina y el útero son menos afectados $\mathrm{y}$ nosotros no encontramos ningún caso, a excepción del relatado más adelante, que no fue lo suficientemente investigado como para sacar una conclusión categórica; pero no dudamos que neoplasias de la vagina, como el quiste de Gardner, no sean tan raras y si no se ven estos casos en la consulta infantil es porque pasan inadvertidos a la madre, puesto que están ocultos dentro de la vagina y no dan ninguna sintomatología llamativa; en general se descubren en una edad más avanzada, al hacer algún examen ginecológico por cualquier otra causa.

La verdadera frecuencia del cáncer ginecológico en las niñas es difícil de determinar. Dargeon (12), autoridad en cancerología pediátrica, afirma que es raro, comparado con la frecuencia en adultos. Claro está, pero en esta era antibiótica y de grandes progresos de la medicina, en donde se hacen toda clase de esfuerzos para establecer un pronto diagnóstico e instituir tratamientos adecuados, a medida que se reducen determinados grupos de enfermedades, toman relieve las de origen neoplásico, cuya incidencia se ve elevada, o mejor dicho, pasan a ocupar los primeros renglones en importancia, lo cual hace necesario emprender su estudio y mejor conocimiento.

Nuevamente llamamos la atención a pediatras y ginecólogos sobre este hecho, para tratar de encontrar precozmente dichas neoplasias y pensar siempre en su diagnóstico ante un flujo, una hemorragia genital, etc., igual cosa ante la presencia de una masa pélvica, interviniendo quirúrgicamente a la mayor brevedad posible.

Este criterio de creer muy raras las neoplasias de las niñas, lo vemos reflejado en los patólogos que dudan mucho al hacer un diagnóstico, precisamente por la edad de la paciente y piden nuevas biopsias o hacen más preparaciones histológicas, para llegar a un diagnóstico positivo correcto. 


\section{CUADRO NUMERO 1}

SITIOS DE LOCALIZACION

\begin{tabular}{|c|c|c|c|}
\hline & \multicolumn{2}{|c|}{ Número de casos } & Porcentaje \\
\hline Genitales externos $\ldots \ldots \ldots \ldots \ldots \ldots$ & 9 & $\ldots \ldots$ & $23.07 \%$ \\
\hline 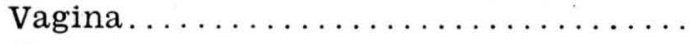 & 0 & $\ldots \ldots$ & $00.00 \%$ \\
\hline Utero .............. & 1 & $\ldots \ldots$ & $2.36 \%$ \\
\hline 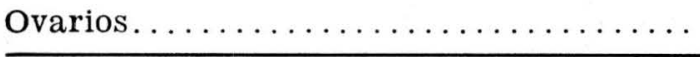 & 29 & $\cdots \cdots$ & $74.35 \%$ \\
\hline TOTAL $\ldots \ldots \ldots \ldots \ldots \ldots$ & 39 & $\ldots$ & $100 \%$ \\
\hline
\end{tabular}

\section{TUMORES DE LOS GENITALES EXTERNOS}

Los genitales externos de la niña son de los sitios en que ordinariamente se asientan lesiones tumorales.

Encontramos en nuestro estuaiio nueve casos de neoplasias de los genitales externos, entre los 30 que componen el total y cuya frecuencia sería del $23.07 \%$.

El hemangioma y el sarcoma son las neoplasias más habituales; el hemangioma ocupa el primer puesto con cinco casos, le sigue el sarcoma con tres: un linfosarcoma, un fibrosarcoma y un caso muy discutido que en un principio se pensó fuera un sarcoma botryodes de la vulva. Hay un caso de papiloma.

Morse (citado por Dargeon), en un repaso exhaustivo de la literatura, sólo pudo encontrar un caso de carcinoma de la vulva en una niña, y el mismo Dargeon (loc. cit.) describe uno de melanoma en el labio mayor izquierdo de una niña que fue atendida en el Memorial Hospital.

Llama la atención que, así como no es raro encontrar el sarcoma en la vulva de las niñas, sea muy raro el carcinoma, el cual es ce alta frecuencia en fases tardías de la vida, y constituye el $10 \%$ según Gurit (15), de todos los cánceres en la mujer.

Angiomas.-Son tumores generalmente benignos, compuestos de vasos neoformados, de crecimiento lento, de desarrollo precoz, congénitos por naturaleza y que, si no se les trata, crecerán lentamente hasta la vida adulta; son clínicamente característicos y fáciles de diagnosticar. La lesión puede ser de color rojo-vino, vio- 


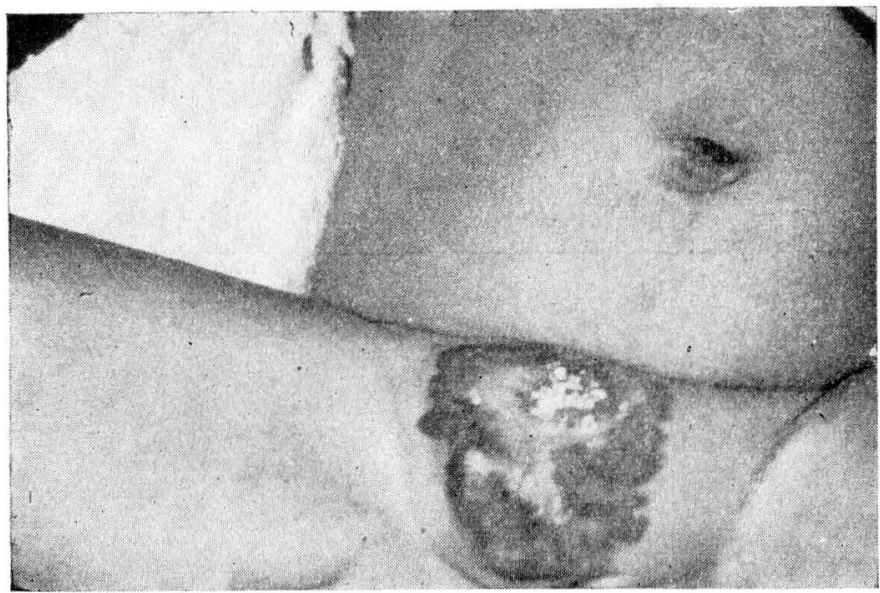

Caso número 5. Hemangioma de los genitales externos (Foto Instituto Nacional de Cancerología).

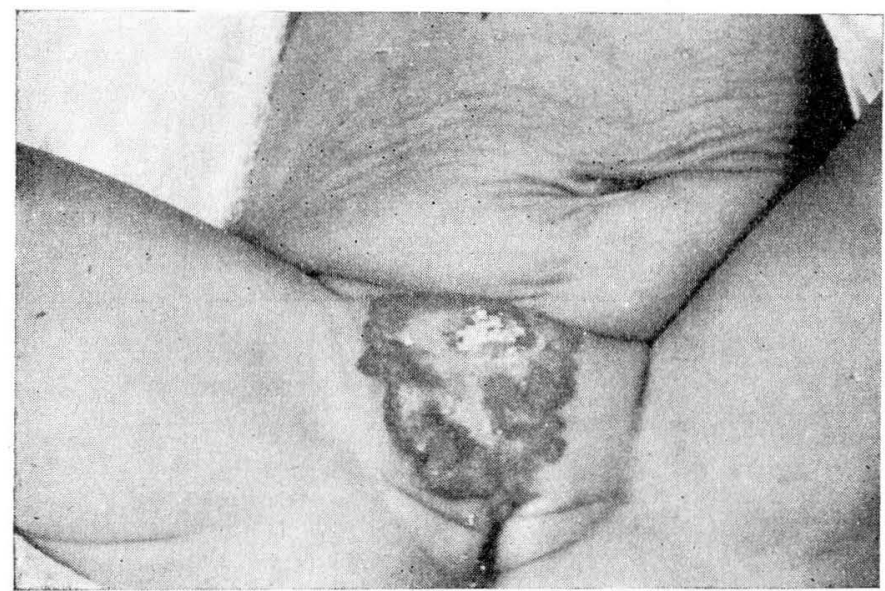

Otro aspecto del caso número 5 (Foto Instituto Nacional de Cancerología)

lácea o azulada, que se ulcera con frecuencia, de consistencia blanda, prominente, indolora, redondeada o irregular, de dimensiones variables y que puede abarcar tanto la piel de la región pubiana como los grandes labios. Histológicamente están caracterizados por la proliferación de vasos sanguíneos o linfáticos, dando el hemangioma o el linfangioma respectivamente, o la combinación de ambos (16). Tienen su origen en secuestros o inclusiones embrionarias de tejido vascular o linfático. Riwert (17) cree que el cre- 


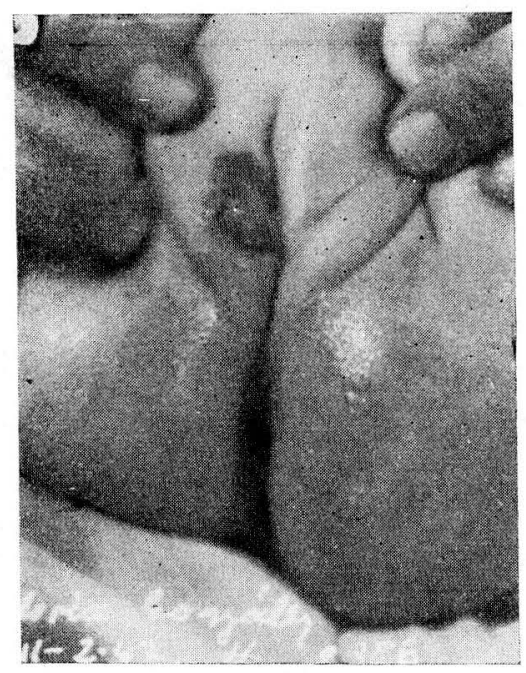

Caso número 8. Hemangioma de los genitales externos (Foto Instituto Nacional de Cancerología).

cimiento tumoral es una extensión o proyección de nuevos vasos en el tejido circundante, más bien que una invasión del proceso tumoral en los vasos sanguíneos. Se han sugerido varias teorías sobre el posible origen. Thoma (18) pensaba que factores mecánicos tienden a excitar nuevos crecimientos de vasos. También se ha supuesto que los angiomas representan hipertrofia simple de ciertos elementos vasculares sin crecimiento neoplásico. Por otra parte se ha insinuado la irritación neurógena, traumática y local, pero la naturaleza congénita del angioma sugiere fuertemente su origen de tumor vascular o linfático descolocado, que ha retenido un cierto poder de crecimiento persistente e irregular (Dargeon, Loc. cit.). Esta es la neoplasia que más afecta a la infancia y en la mujer es dos veces más frecuente que en el hombre. Tiene predilección por la cabeza y alrededor de los orificios naturales. Por su estructura, se describen principalmente dos variedades clínicas: el hemangioma simple, nevo vascular (telangectasia) o "mancha de vino tinto" y el cavernoso, que semeja por su estructura los cuerpos cavernosos del pene, constituído por las neoformaciones vasculares muy dilatadas y los tabiques de los tejidos conectivos, algo tenues. Puede haber numerosos tipos de transición entre estas dos modalidades principales (19). Como complicación se puede presentar la ulceración y hemorragia consecuente. Las perturbaciores funcionales son debidas a su localización, por su crecimiento. 
E:l tratamiento se hace con radium, rayos $\mathrm{X}$, o cirugía. Algunas veces se han usado rayos ultravioleta, nieve carbónica, electrocoagulación, etc., con resultados variables. Por regla general el linfangioma es radioresistente. Por otra parte, los angiomas de los genitales externos de la niña, se prefieren tratar por métodos quirúrgicos, ya que las irradiaciones son peligrosas por la proximidad de los ovarios. El tratamiento es por lo común plenamente satisfactorio.

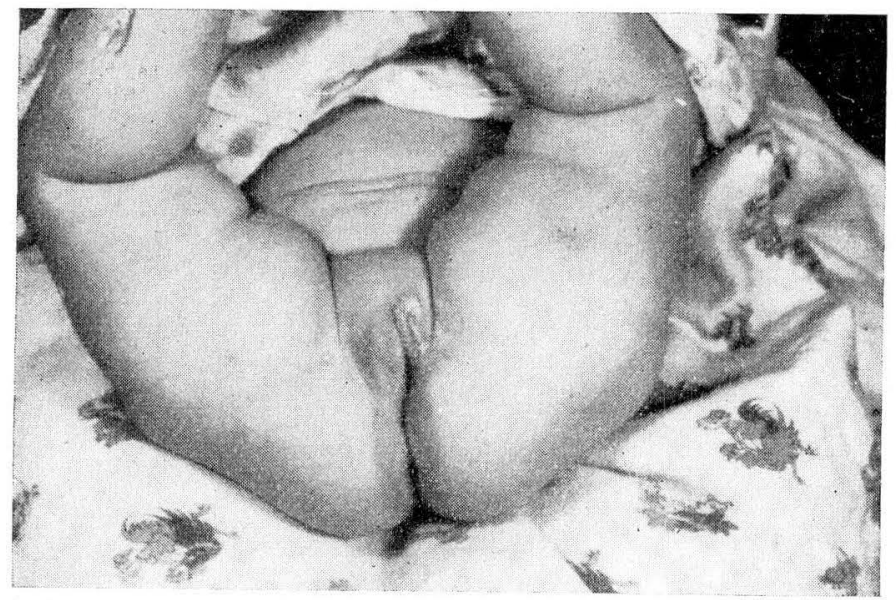

Caso número 7. Hemangio-endotelioma de los genitales externos (Foto Instituto Nacional de Cancerología).

El nemangio-endotelioma está constituído por un gran número de pequeños vasos tapizados por endotello hipertrófico e hiperplásico; la luz de los vasos se estrecha, pero la proliferación endotelial puede llegar a obliterarla y producir espesos grupos celulares (Ewing. Op. cit).

En todos nuestros casos la lesión era congénita, en tres había fenómenos de ulceración; en todos estaban interesados los grancles labios: en tres, el labio mayor derecho, en uno, el izquierdo y en el otro caso, ambos labios. En ningún caso hubo antecedentes traumáticos, familiares o de otra naturaleza. El caso de menor edad fue de un mes y el de mayor edad de ocho meses. A dos casos se les hizo tratamiento quirúrgico con resultados satisfactorios, dos no volvieron después de diagnosticada la lesión y el otro caso fue enviado al Hospital de La Misericordia por malas condiciones generales y se pierde de vista. 
Paxilomas. - Son tumores de curso ordinariamente benigno; en la mayoría de los casos la capacidad proliferativa no es acentuada y deriva del epitelio pavimentoso, con tejido de soporte de distribución normal.

En el caso nuestro, había tres lesiones verrugosas de tipo vulgar en los labios mayor y menor izquierdos. También había en los miembros superiores. La serologia fue negativa. Se extirparon las lesiones y se hizo electrocoagulación, con resultados satisfactorios.

Linfo y fibrosarcomas. - En general son todos aquellos tumores en cuya constitución entran células redondas sarcomatosas, que algunos autores llaman "sarcomas de células redondas", y, si el tipo de estas células es de tamaño grande, se llamará "linfosarcoma linfoblástico"; si son pequeñas las células redondas se dirá "linfosarcoma linfocítico". Cuando el sarcoma está constituído por células fusiformes se le denominará "fibrosarcoma". Lo más común es que se presenten en forma aguda y se desarrollen rápidamente $(20)$.

Queremos hacer referencia especial al llamado "sarcoma botryodes" por atraernos poderosamente la atención el caso que aquí relatamos y el cual vimos e intervinimos de cerca.

El término descriptivo botryodes $(\mathrm{gr}$. botrys $=$ racimo de uva) fue aplicado por primera vez por Pfannenstiel en 1892; sin embargo, el primer caso fue descrito por Wagner (21) en 1854 y

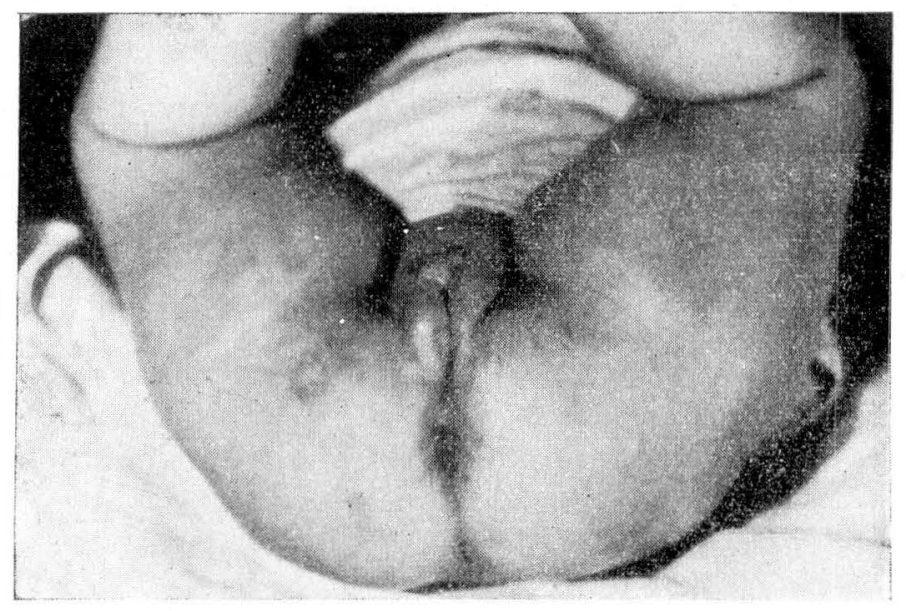

Caso número 1. Diagnosticado como Sarcoma Botryoides de la vulva (Foto Instituto Nacional de Cancerología). 
Spiegelberg (22) describió la lesión en 1897 como "sarcoma colliuteri hidropicum papillare". Se han descrito aproximadamente, unos 100 casos en la literatura mundial; es un tumor ginecológico raro, de alta malignidad, que se puede presentar en mujeres adultas. Spademan (23) publica un caso en una mujer de cincuenta y ocho años, pero la mayoría de los casos relatados en la literatura se refieren a la infancia. La vagina, el cuello y el cilerpo uterino son 105 sitios de predilección. El caso nuestro es de la vulva. Simpson (24) cree que el sarcoma botryodes del útero es más frecuente en edades avanzadas (cuarenta y cinco a sesenta y cinco años) y el de la vagina y vulva lo es en niñas. Macroscópicamente se caracteriza por una masa de apariencia gruesa en forma de uva, que aparece como un pólipo, a veces edematoso y de coloración rojiza como de carne (sobre todo cuando está cubierto, como en vagina, cuello y cuerpo), friable, porque puede tener zonas de necrosis y hemorragias. Sangra con facilidad y la hemorragia puede ser sero-sanguinolenta y con mal olor por la infección secundaria. Al seccionarlos hay cavidades quísticas con hemorragia, necrosis y supuración. El tipo más común de tejido es un estroma de apariencia mixomatosa fioja.

Microscópicamente se observa, en primer lugar, un epitelio que recubre el tumor y propio del sitio donde se localice la lesión; luego una compacta capa sub-epitelial de elementos conjuntivos redondos o fusiformes. El centro del tumor está cons-

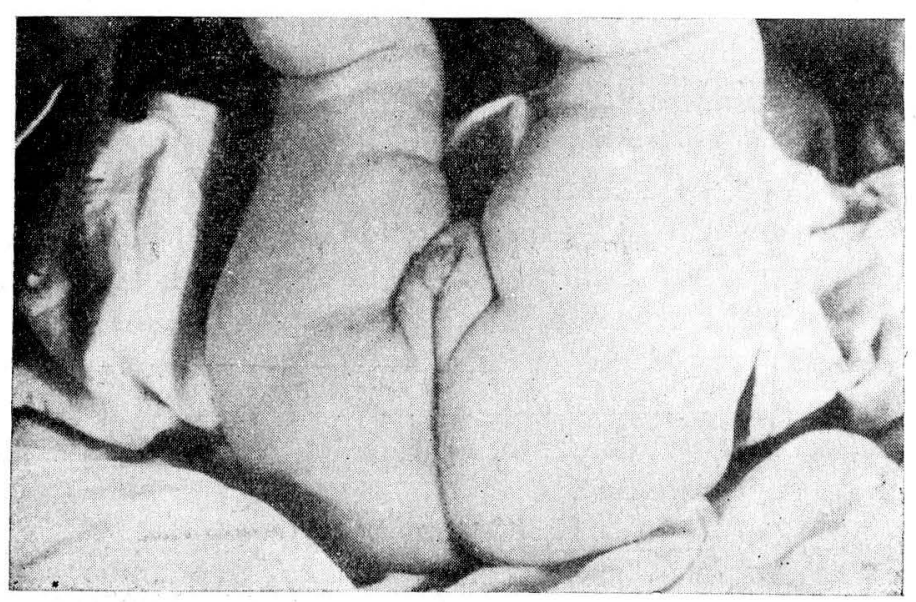

Otro aspecto del caso número 1 (Foto Instituto Nacional de Cancerología). 
tituído por células fusiformes aisladas o agrupadas en fascículos con núcleos aislados de disposición en rosario; otras agrupaciones semejan células gigantes; un estroma de células estrelladas de apariencia mixomatosa, y finalmente, una irrigación discreta por vasos de constitución completa y aspecto adulto. Esto es lo que se observa con las coloraciones corrientes, no hay muchas mitosis, es un cuadro histológico "tranquilo", dice Conill-Montobbio (25). Pero, haciendo coloraciones argénticas especiales, afirma el mismo autor, que observando preferentemente la capła sub-epitelial, se encontrará la clave del diagnóstico: las aparentes células conjuntivas no son otra cosa que típicos rabdomioblastos en fase muy precoz, con estriaciones longitudinales, y con estriaciones transversales, propias de la fibra muscular adulta. Las tales células gigantes son en realidad aglomeración de rabdomioblastos. En cuanto al estroma, son simples elementos conectivos jóvenes.

La histogénesis ha sido explicada por Mc Farlan (26) como una dislocación accidental de células embrionales residuales y su inclusión entre otras que retienen sus posiciones normales en la formación del aparato urogenital. Glas (27) presenta 58 casos, de los cuales sólo cuatro por debajo de los diez años y 10 por debajo de los veinte. Richmond (28) publica un caso de sarcoma botryoides del cuello uterino en una niña de quince meses; la familia afirmaba que la masa tumoral que asomaba por la vagina habia sido observada desde el nacimiento. Fue operada, haciéndole histerectomía radical y llevándose gran parte de vagina; controlada a los dieciocho meses de la intervención, vivía sin señales de enfermedad residual. Llama la atención en este caso el hecho aparente de ser congénito y que, al control practicado, estuviese libre de enfermedad puesto que, se afirma, no obstante no dar metástasis a distancia, su curso es altamente maligno y recurrente. El mismo autor no es optimista, porque la experiencia ha señalado que todos los casos son recurrentes a pesar de no existir metástasis o invasión e independientemente del tratamiento empleado, ya sea cirugía, radium o rayos $\mathrm{X}$. Sin embargo, un caso operado en 1947 -el de Ulfelder (29) - vive aún, y Stoeckel (30) comenta el caso de von Schuchardt con diez años de supervivencia.

Sobre este tema del sarcoma botryoides, algunos otros autores se han ocupado, tales como Sadler (31), Keller (32), etc. La última publicación, conocida por nosotros, sobre el sarcoma bo- 
tryoides, es la de Ober y Palmer (33) de octubre de 1953, donde comunican dos casos en adultos, uno en la vagina y otro en la vulva.

En cuanto al caso relatado aquí, no ha transcurrido un tiempo prudencial para juzgarlo. Fue tratado después de extirpación-biopsia de la lesión, con radio-punción vulvar y linfadenectomía bilateral, sin evidencia de metástasis. En junta de decisiones se discutió ampliamente el tratamiento a seguir, pero razones de orden humano se tuvieron también en cuenta para no practicar una operación tan radical como la vulvectomía. En el último control practicado a los cinco meses de haberse iniciado la enfermedad, no se encontraron señales de recurrencia local o indicios de metástasis. Consultado este caso con un connotado cancerólogo roentgenterapista extranjero, de visita en nuestro pa's, comentó que "aualquier tratamiento era bueno, pues se moriría de todos modos".

El caso de linfosarcoma, se presentó en una niña de ocho meses. La lesión se inició en la región fronto-parietal izquierda, la cual se diagnosticó originalmente como fibrosarcoma, pero por la radiosensibilidad se pensó en linfosarcoma; revisadas las placas histológicas se confirmó este último diagnóstico. A los tres meses de haber comenzado la enfermedad, apareció una lesión de la misma naturaleza en el labio mayor izquierdo de la vulva. Fue tratada quirúrgicamente y con radium. La historia no registra los resultados ulteriores.

En el caso de fibrosarcoma, la lesión estaba localizada en el hímen. La enfermedad fue progresando a pesar de haber sido intervenida dos veces, y evolucionó en siete meses hasta la muerte. También se le hizo radioterapia, previa implantación de ovarios en axila, y la cual hubo que suspender por las malas condiciones generales de la enferma.

Otros tipos de neoplasias pueden existir en los genitales externos de la niña, pero sólo nos limitamos a comentar aquellos tipos que hemos encontrado. 


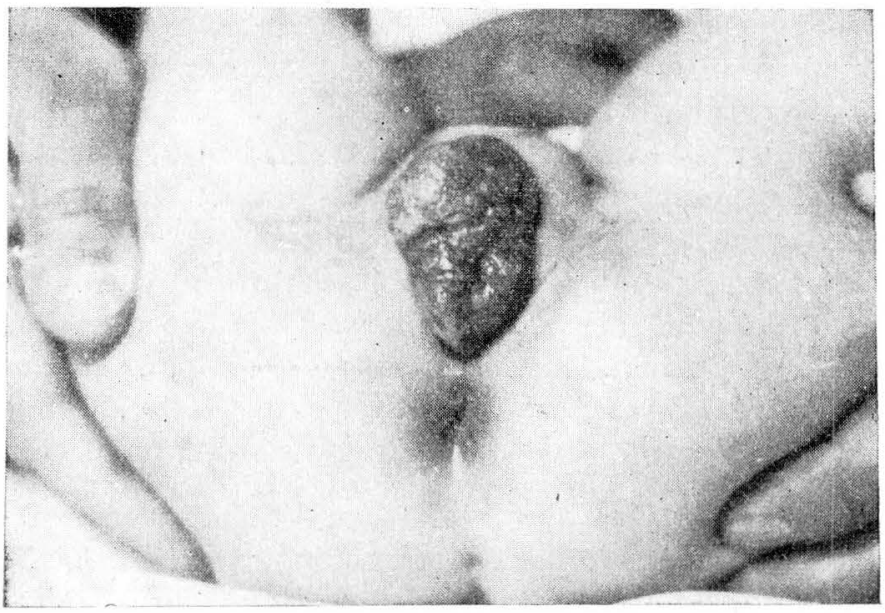

Caso número 2. Fibrosarcoma del himen (Antes) (Foto doctor Mario Gaitán).

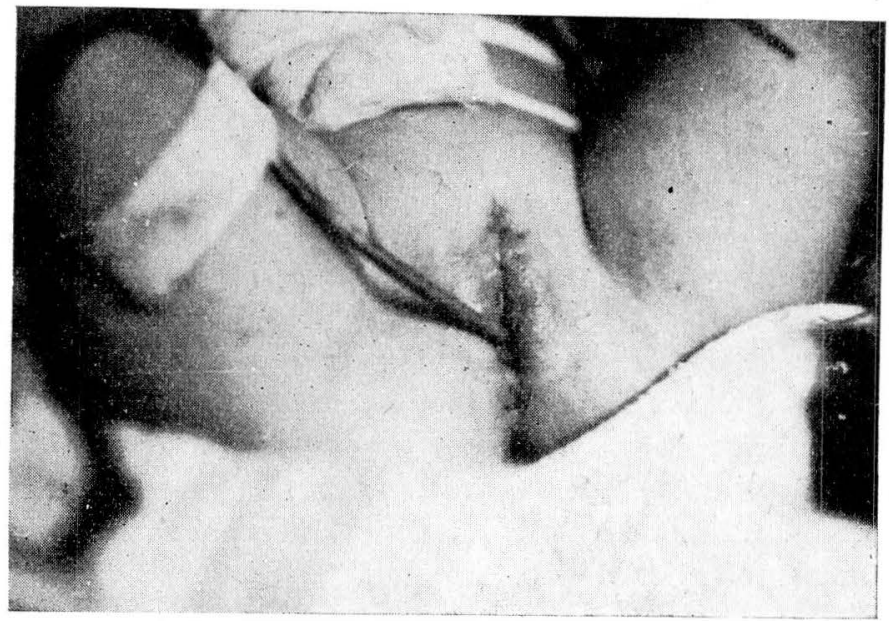

Caso número 2. Fibrosarcoma del himen (Después) (Foto doctor Mario Gaitán). 


\title{
CUADRO NUMERO 2
}

\author{
GENITALES EXTERNOS
}

\begin{tabular}{|c|c|c|}
\hline $\mathrm{N}$ & Número de casos & Porcentaje \\
\hline Hemangiomas $\ldots \ldots \ldots \ldots \ldots \ldots \ldots$ & $\ldots \ldots$ & $55.55 \%$ \\
\hline 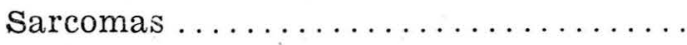 & $\ldots \ldots$ & $33.33 \%$ \\
\hline 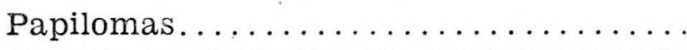 & $\ldots \ldots$ & $11.11 \%$ \\
\hline$\ldots \ldots \ldots \ldots \ldots$ & 9 & $100 \%$ \\
\hline
\end{tabular}

\section{TUMORES DE LA VAGINA}

Aun cuando nosotros no encontramos ningún caso, en la vagina de la niña ocasionalmente pueden desarrollarse lesiones tumorales. Ya hemos explicado, que si el quiste de Gardner, incidentalmente se puede hallar en la mujer adulta en el curso de un examen ginecológico practicado por algún otro motivo, es mucho más lógico suponer su existencia desde la infancia, pero no se hace su diagnóstico, ya que no se visualiza, ni produce ninguna sintomatología aparente que induzca a consultar. Recordamos un caso de una mujer de veintidos años que ingresó por alguna otra ginecopatía al Servicio de Ginecología del Hospital de San Juan de Dios, descubrimos al tacto una pequeña masa tumoral, blanda, en la cara anterior de la vagina y con un diagnóstico clínico de quiste de Gardner, la operamos y posteriormente la anatomía-patológica confirmó ese diagnóstico. De manera pues, hay que tener presente la posible existencia de tal lesión.

La mayor parte de casos de sarcoma botryodes publicados, han sido en la vagina. Ya hemos visto como Simpson (loc. cit.) considera que en la vagina de las niñas es de más frecuente ocurrencia este sarcoma, así como lo es el mismo en el útero de la mujer adulta. Debe sospecharse cuando haya una persistente hemorragia vaginal en una niña de cualquier edad, desde la infancia hasta la adolescencia.

En cuanto al carcinoma de la vagina, entidad rara en la mujer adulta $-0.43 \%$ de la totalidad de los carcinomas de la mujer según Williams (34) - , lo es aún más en la niña y Ewing 
(Op. cit.) llega a afirmar que los casos citados en niñas son de naturaleza dudosa. Winckel (35) cita siete casos de carcinoma de la vulva, encontrados entre los diez y los treinta años, pero rio dice cuántos casos corresponderían a la niñez. Baldwin (36) informa de un caso de carcinoma escamoso primario de la vagina en una niña de catorce años. Entre 905 casos de cáncer de la vulva, vagina, cuello y cuerpo uterino, demostrados por estudios microscópicos en el Hospital de la Universidad de Michigan, este fue el único caso de cáncer de los genitales femeninos que se presentó en una paciente menor de veinte años.

Schauffler (37) cita un caso de adenocarcinoma vaginal en una niña de tres años, que se originó posiblemente en los tejidos profundos de la glándula de Barthoin izquierda; fue la evolución altamente maligna y la niña murió.

De este apresurado recorrido por la literatura médica, se deduce sin embargo, la muy baja frecuencia de afecciones tumorales en la vagina de la niña, que constituyen verdaderos raros hallazgos de la ginecología infantil.

\section{TUMORES DEL UTERO.- CUELLO Y CUERPO.}

Al igual que la vagina, el útero de las niñas tampoco es órgano que tenga afinidad por las neoplasias.

E! carcinoma del cuello uterino, de tan alta frecuencia entre todos los cánceres del organismo - y casi siempre de la mujer que ya ha dado hijos- no es, con mucho, frecuente en la infancia. Sin embargo a todo hay su excepción y la literatura médica registra algunos casos. En una niña de diez años, Morse (op. cit) comunica la presencia de un adenocarcinoma del cuello, de evolución altamente maligna y en un minucioso repaso de la literatura pertinente, sólo encontró ocho casos más de neoplasias epiteliales que afectaban el cuello o el cuerpo uterino. Detalladamente analiza y discute estos casos para concluir, que en tres casos el diagnóstico debe ser aceptado con reservas, en dos el informe fue incompleto y en sólo tres de los ocho casos, la evidencia clínica e histológica, confirmarían el diagnóstico de carcinoma.

Más recientemente, otros autores dan cuenta de adenocarcinomas del cuello en niñas de dieciséis años -Dargeon, Glas y Ludwin comunican cada uno un caso- (loc, cit.). En una niña de ventidós meses, Schffey y Grawford (38) presentan un ade- 
nocarcinoma del cuello. Bonner (39) en una niña judía de trece años, halló un adenocarcinoma papilar del cuello, aunque es bien sabido que las mujeres de raza judía son menos propensas a desarrollar carcinomas del cuello uterino, que las mujeres de otras razas.

Según informe personal de López Escobar, en el Instituto Nacional de Cancerología, hubo en el año de 1949, un caso de carcinoma exofítico del cuello, estado III, en una prostituta de quince años de edad y confirmado histológicamente. No pudimos encontrar la historia ni el protocolo de este caso.

En 1932, Gilbert (40), presentó un caso de carcinoma del cuerpo uterino en una niña de once años y como para que no quedare duda, las placas histológicas fueron revisadas y confirmado el diagnóstico por varias autoridades en la materia, de esa época. En ese entonces el autor mencionado encontró en la literatura, noticias de sólo 5 casos más de carcinoma del cuerpo viterino en pacientes menores de quince años.

Para 1935, Lockhart (41) informaba de un caso de adenocarcinoma del cuerpo uterino, en una niña de dos años y tres meses, que parecía insólito y extraordinariamente raro por la edad.

Habiamos dicho antes (Simpson, Loc. cit.), que el sarcoma botryoides también se puede encontrar en el útero, pero más frecuentemente en edad avanzada y que en las niñas sería más común en la vagina.

En el informe de Glass (Op. cit.) sobre sarcoma botryoides, vimos que sólo habían cuatro casos del cuerpo uterino en niñas menores de diez años y diez casos por debajo de veinte años; también vimos el caso publicado en 1951 por Richmond (Op. cit.), en una niña de quince meses, con sarcoma botryoides del cuello uterino, al parecer congénito.

Dentro de esta excepcional incidencia de tumores del útero en las niñas, como hecho interesante, el sarcoma no ocurre más que el carcinoma.

James (42), de Inglaterra, publica recientemente -año de 1951-, un caso de un tumor polipoide benigno, en una niña de tres años, que él considera único al no encontrar otro semejante, en una búsqueda en la literatura médica de los últimos veinte años.

En el Hospital de La Misericordia hubo un caso que, desafortunadamente, no fue estudiado en forma adecuada. Por haber muerto en el funesto 9 de abril de 1948, no se le pudo practicar 
autopsia. Podría ser uno de los raros casos en la literatura médica de carcinoma genital a tan corta edad, puesto que se trata c.e una niña de dos y medio años. No es posible hacer una afirmación categórica sobre su verdadero diagnóstico. En ningún momento se practicó examen ginecológico o urológico.

Sin precisar la fecha, la enferma "expulsa un fragmento de tejido por los órganos genitales", el cual se envía para estudio histopatológico. El informe correspondiente dice: "Descripción macroscópica: un fragmento de tejido blando de dimensiones $3 \times 2$ x 1 centímetro, cuyo aspecto recuerda al de un coágulo de sangre y de fibrina. Descripción histológica: el examen histológico muestra un tumor de tipo maligno, al parecer epitelial (adenocarcinoma?), cuyo órgano de origen no es posible precisar. Se recomienda practicar un examen completo del aparato génito-urinario y ano-rectal, como también linfático (ganglios) y, si es posible, enviar más material para examen".

La enferma muere el 9 de abril de 1948, sin que la historia consigne ningún detalle sobre el particular o evolución de la enfermedad. Sólo se anota que la enferma fue tratada con "sueros, cesinfectantes, penicilina y tónicos".

\section{TUMORES DE LOS ANEXOS: OVARIOS, TROMPAS, PARAOVARIOS, ETC.}

Los tumores del ovario, sin la menor duda, constituyen el capítulo más extenso - por ser los más frecuentes- dentro de las reoplasias genitales de la niña. Todos los autores coinciden en esto y el número de nuestros hallazgos -29 casos en 39- así lo confirma, con una frecuencia de $74,35 \%$.

No encontramos tumores de trompas, paraovariosa, etc., ni vimos en la bibliografía consultada ningún caso.

Harned (43) señala a los tumores del ovario una frecuencia en cerca del $1 \%$ de todos los tumores infantiles. Se puede encontrar prácticamente toda la gama de tumores que se ven en el adulto: quistes dermoides, quistes simples de retención, carcinomas, sarcomas, etc., esto debido probablemente a que el ovario "presenta un amplio campo estructural, una mayor variación individual y unas bases embriológicas e histogenéticas más complejas que las de cualquier otro órgano" (Ewing, op. cit.).

En todas las edades se pueden observar los tumores del ovario, pero son más frecuentes en el período de vida sexual (vein- 
ticinco a treinta y cinco años). Algunos pueden ser congénitos, como el quiste dermoide o el quiste simple, tal el caso que relatamos aquí del doctor Acosta Bendek, de Barranquilla.

Dorand (44) ha descrito un sarcoma y un cistadenoma fetales y Lonneberg (45) un sarcoma bilateral. Weil (46) recopiló 24 casos en niñas menores de cinco años y 60 en menores de diez años. Hubert (47) reunió 175 casos de tumores del ovario en la infancia. Hall y Bagby (48), al revisar las estadísticas de treinta años (1908-1938) del Bernard Free Skin and Cancer Hospital, de Saint Louis, encontraron que de 6 casos de cáncer del tracto genital femenino, uno era un carcinoma coloide del ovario en una paciente de quince años.

Lesh (49), en un trabajo publicado en 1944, encontró 99 casos de quistes del ovario en niñas, al revisar tanto la literatura norteamericana como la inglesa; más del 50\% eran niñas que se aproximaban a la nubilidad.

En años más recientes se han publicado varios casos, o series cie casos, de lesiones ováricas malignas en niñas (Downes, Hunt, Simon, Gland y Goldstein, Anderson y Sheldon, Levi, Lamman, etc.), los cuales pueden ocurrir a muy temprana edad, como el caso de Hunt (50), que tenía diecisiete meses, y 16 de los casos cie Downes (51) fueron menores de diez años.

Ewing (Loc. cit.), hace notar el predominio de teratomas, carcinomas y otros tumores malignos, en los primeros años de la vida.

En contra de lo que piensa Pack (Op. cit.), Kelly (52) cree en la alta mortalidad operatoria de la infancia.

Es notable que las neoplasias ováricas malignas en adultos, no formen un alto porcentaje en relación a todos los tumores malignos de los genitales femeninos. En el Memorial Hospital, Dargeon (54) es de opinión similar, pero hace hincapié en decir que el cáncer del ovario es la forma más frecuente de neoplasia maligna en el aparato genital de las niñas.

En cuanto a teratomas malignos se refiere, Lisa (55) cuenta ciue, según las clasificaciones de Wakely y Witzbenger y Agerty, figuraban hasta mediados de 1949, en sólo las dos primeras décadas de la vida, alrededor de 150 casos auténticos, incluyendo aquellos casos publicados como dermoides, porque según el mismo autor, aunque histológicamente benignos, los dermoides se pueden manifestar en los niños como malignos. Posteriormente se publicaron 24 casos más, de los cuales 10 eran benignos y 14 ma- 
lignos; de estos 24 casos, la tercera parte aproximadamente mostró evidencia cierta de malignidad.

Lisa (Loc. cit.) reconoce que en publicaciones recientes se encuentran con mayor frecuencia casos de teratomas malignos, lo cual atribuye, como también lo ha sugerido Pownes (56), a que se hacen exámenes histológicos más completos y rígidos.

Sobre disgerminomas, Pedowitz y Graycel (57) reportan 5 casos en edad prepuberal y la paciente más joven era una niña ce seis años.

Como sintomas de los tumores ováricos, en términos generales son los siguientes: 10 Tumoración pélvico-abdominal; $2^{\text {o }}$ Efectos mecánicos sobre las vísceras vecinas (sistema gastrointestinal o urinario, consistentes en constipación o diarrea, vómitos, polaquiuria, disuria, tenesmos, etc.) ; 3 ? Cambios sexuales precoces, en algunos tipos de tumores, y $4^{\circ}$ La torsión, que es más bien una complicación, pero que habitualmente es por ella, por su sintomotología dramática, por lo que llega a descubrirse la presencia cie un tumor ovárico en una niña, como sucedió en 7 casos de nuestra serie; uno era una niña de catorce meses y los otros, de siete, doce y trece años de edad. Otras complicaciones han sido señaladas, tales como infección, ruptura, malignización y hemorragia.

Los quistes simples de retención no son propiamente formaciones neoplásicas, pero se incluyen en este trabajo, primero, por el hecho importante de encontrarse en niñas de corta edad, y segundo, por la estrecha relación que guardạn con los verdaderos tumores ováricos.

Encontramos 5 casos de este tipo, uno en una niña recién nacida - caso de Acosta Bendek, de Barranquilla_, y todos los otros en la primera mitad de la segunda década de la vida.

El cistadenoma, el tumor ovárico más trecuente de la mujer aduita, es una hiperproducción e hiperplasia del tejido germinal del ovario con proliferación celular y desorganización de la estructura ovárica y formación de celdas que se llenan de un líquido que puede ser seroso o pseudomucoso, carácter que da el nombre al tumor, de seroso o pseudomucoso (Aparicio, loc. cit.). Su frecuencia, estimada en un $21 \%$, es sensiblemente igual en ambas variedades. Parece que el pseudomucoso es el tumor que alcanza al mayor tamaño del cuerpo humano. Histológicamente se caracterizan por el epitelio de la pared tumoral, que puede ser cuboide o columnar bajo, piuriestratificado por lo general, sim- 
ple o con tendencia a la formación de papilas, que son menos frecuentes en el pseudomucoso. Las células, en muchos casos, son ciliadas y bastante parecidas a las del epitelio tubárico.

Encontramos dos casos en nuestra serie, uno el caso de clientela particular de López E., que era un cistadenoma seroso en una niña de doce años, con fenómenos de torsión; el otro en San Juan de Dios, en una niña de catorce años, se hizo diagnóstico operatorio de cistadenoma pseudomucoso y la anatomía patológica informó que se trataba de un adenoma papilar quistico, que, como es sabido, es una variedad del cistadenoma seroso con tendencia a la malignización. En otro caso de diagnóstico clínico de cistadenoma pseudomucoso, fue refutado por la anatomía patológica; se tiataba de un teratoma benigno, según concepto del patólogo A. P. Stout.

Los tumores malignos de origen epitelial son el carcinoma prímitivo y el carcinoma secundario o metastásico. De ambos tipos de neoplasias encontramos casos.

La sintomatología inicial de estos tumores es vaga y obscura. Parece que tampoco producen alteraciones en la esfera menstrual; en etapas más avanzadas hay ascitis y algunas veces derrame pleural. El adenocarcinoma es la variedad más común. La frecuencia del carcinoma ovárico primitivo ha sido señalada en un $10 \%$ de todas las neoplasias ováricas. El llamado tumor de Krukemberg, descrito por este autor en 1896 como ordinariamente bilateral, de considerables dimensiones, que conserva la forma de la glándula, que se puede presentar a cualquier edad, de curso fatal por propagación y recidiva y caracterizado histológicamente por las denominadas células en forma de sello -originadas en los cambios mucoides del epitelio; es un carcinoma secundario, metastásico, generalmente del tracto genital o gastrointestinal (estómago primordialmente). El mismo Krukemberg no cayó en la cuenta de que se trataba de un carcinoma metastásico. Sin embargo, se admite que existen tumores primitivos del ovario de tipo Krukemberg, es decir, con todas las características macro y microscópicas descritas por este autor. A pesar de que Goldstein (59) afirma que el carcinoma primitivo es raro en la niñez y de que Sager (60), de 42.864 admisiones en el Children's Hospital de Washington, sólo encontró uno en una niña de nueve meses, nosotros creemos que la frecuencia es alta - por lo menos en nuestro medio-, hecho que deducimos de nuestro estudio, ya que de 55.000 informes histopatológicos revisados, aproximada- 
mente encontramos 6 casos de carcinoma primitivo del ovario en niñas y un caso de carcinoma secundario en una paciente de dieciséis años. Comparado esto con los datos de Sager, resalta palpablemente la mayor frecuencia en nuestra serie. En este informe, la enferma de menor edad fue de diez años, y la de mayor, de dieciséis años. Tres casos en niñas de doce años y uno en una de catorce años. De 4 cuatro casos, sólo se tienen los datos de la anatomía patológica, y por lo tanto se desconoce su evolución y pronóstico. Los otros dos casos murieron a los dos y tres meses de iniciada la enfermedad, respectivamente. En uno se hizo el diagnóstico a la autopsia, y el otro fue operado, muriendo nueve días después.

Del caso del carcinoma secundario tampoco se conocen su evolución y pronóstico.

De las neoplasias conjuntivas, hallamos un caso de tumor conjuntivo maligno; un sarcoma fusocelular del ovario en una niña de cuatro años. Los datos que tenemos son los de la anatomía patológica y únicamente sabemos que tenía un diagnóstico clínico de sarcoma del riñón.

El sarcoma del ovario es un tumor altamente maligno y muy raro. Tiene tendencia a ocurrir en una edad más avanzada que el carcinoma. Su frecuencia en relación con los tumores malignos epiteliales, ha sido señalada en el uno por cuarenta de todas las neoplasias del ovario (61), y otros autores señalan el 0,8\% (62). Histológicamente es un tumor semi-encapsulado, de consistencia sólida, carnoso al corte y cuyo aspecto microscópico adopta las características del sarcoma de cualquier otro órgano, encontrándose las variedades de sarcomas de células redondas, mixto o indiferenciado, fibrosarcoma, linfosarcoma, etc.

Los foliculomas del ovario pueden tener una evolución maligna; se desarrollan a expensas de elementos granulosos o tecales, que darán tumores de células de la granulosa o tumores de células tecales. Novak (63) ha sugerido un posible origen mesenquimatoso. Se presentan en todas las edades, inclusive en el climaterio, aunque el derivado de células de la teca no ha sido descrito por debajo de los quince años. Estos tumores no son muy frecuentes; entre nosotros se han descrito algunos casos. Producen marcados efectos estrogénicos, variando la sintomatología, como es natural, si se trata de niñas, mujeres adultas o en la edad del climaterio. Lo que más llama la atención en las niñas, As la producción de reglas anovulatorias, fuera de los signos es- 
trogénicos impropios de esa edad; en la mujer adulta se manifiesta por trastornos de la menstruación, y en las climatéricas por la hiperplasia endometrial y las hemorragias.

Nosotros informamos de un caso de una niña de seis años. De Pereira fue enviada la pieza quirúrgica - el ovario izquierdofara su estudio histológico en el Instituto Nacional de Radium (año de 1939). En el protocolo relativo a este caso figura el diagróstico de "epitelioma folicular". Esta placa la hicimos revisar del doctor Lichtenberger, quien hizo diagnóstico de tumor de células de la granulosa.

De arrenoblastoma se citan sólo unos 97 casos en la literatura mundial, y entre nosotros parece que únicamente se han publicado dos: uno en Medellín, en "Antioquia Médica" y otro en Bogotá, el caso relatado por López Escobar (64). Recordamos que es un tumor sólido, de efecto masculinizante y que produce alteraciones de la mestruación, como oligomenorreas, amenorreas y a veces también esterilidad.

El disgerminoma carece de efectos hormonales y aunque morfológicamente maligno, se ha considerado hasta ahora de benignidad relativa (Pedowitz, op. cit.); otros creen que es de gran malignidad. El nombre se lo dio Robert Meyer y es idéntico al "seninoma" testicular descrito por Chevassu en 1906, quien lo separó de los sarcomas. Se puede presentar en edades bastante tempranas y la estructura y caracteres clínicos son tal vez, de lo más típico en todas las neoplasias del ovario. Es radiosensible y generalmente unilateral. Puede alcanzar gran tamaño. Es un tumor sólido, de consistencia elástica y puede presentar fenómenos de necrosis y hemorragia. Algunos creen que la herencia juega algún papel en este tumor. Por otra parte, la teoría de Meyer es la más aceptada: "Está compuesto de células sexualmente indiferenciadas, semejantes a las que se ven en el período no diferenciado de la evolución gonadal, circunstancia que permite explicar el por qué se encuentran tumores idénticos en el hombre con el nombre de seminoma" (citado por Pedowitz), y este último autor supone que deriva de las células del mesenquima gonadal primitivo, el cual tiene manifiesta posibilidad de diferenciarse en cualquier dirección en la mujer. Si ocurre con tipo masculino, dará el arrenoblastoma; si con tipo femenino, el tecoma o tumor de células de la granulosa; si de aspecto neutro, el disgerminoma. En el hombre, el seminoma. El mismo Pedowitz relata un caso de dis- 
germinoma en una niña de seis años y Ziegler (Loc. cit.) publica un caso en un feto de treinta semanas.

$\mathrm{Su}$ frecuencia parece ser de aproximadamente un $3 \%$ de los tumores malignos primitivos del ovario, y existen alrededor de 200 a 300 casos publicados.

Entre nosotros se han descrito unos 3 casos en adultos. En nuestra serie presentamos 2 casos de disgerminoma del ovario, primeros y unicos hasta ahora, que aparecen en la literatura colombiana, sobre este tipo de tumores en niñas, $y$ de cuya existencia, por primera vez, se informa en este trabajo.

Se trata de pacientes de diez y catorce años respectivamente. Fn ambos, sólo disponemos del informe anatomo-patológico. El primer caso es de una niña intervenida en Cartagena por el doctor Napoleón Franco Pareja, se extrajo un tumor del ovario izquierdo y se envió al Instituto Nacional de Radium. Esto fue en el año de 1938. El reporte anatomo-patológico dice: "Seminoma del ovario". Esta placa la hicimos revisar por el doctor Lichtenberger, quien diagnosticó más exactamente: "Disgerminoma del ovario". En el otro caso se había diagnosticado: carcinoma embrionario; también hicimos revisar cuidadosamente esta placa del doctor Lichtenberger, quien conceptuó se trataba de un disgerminoma del ovario.

De los 3 casos descritos en adultos, uno es el de Pinto (65), del cual habla en su tesis de grado en 1932; el otro, publicado por Amorocho y Lichtenberger (66), y el tercero fue un hallazgo de éste último, al revisar láminas del Hospital de San Juan de Dios, correspondientes al año de 1944. Era una mujer adulta de ventitrés años de edad; protocolo número 3.768. El caso nuestro es uno niña de catorce años, en 1950, protocolo número 09.255.

Este año se ha discutido en el Instituto Nacional de Cancerologia, un caso de autopsia, sobre posible disgerminoma, también en una mujer adulta y que hasta el momento no se ha podido aclarar.

Los teratomas se originan de las tres hojillas embriológicas; serán benignos si están formados de tejidos maduros y se les denomina usualmente quistes dermoides; si de tejidos inmaduros o embrionarios, serán malignos y se les llamará teratomas sólidos o teratoma propiamente dicho. Por regla general no producen alteraciones menstruales y casi siempre son unilaterales; dolorosos a la presión, no alcanzan gran tamaño. Ambos son frecuentes en edades juveniles y de nuestro trabajo se desprende que el quiste 
Ciermoide es el tumor genital más frecuente en la nĩnez, puesto que encontramos diez casos, de donde se deduce una frecuencia del $25,64 \%$ de todas las ginecopatías tumorales en la niñez - halladas por nosotros-, y un $34.48 \%$ de los tumores del ovario.

Tenemos un caso de teratoma maligno, diagnosticado, al revisar las placas histológicas, el cual no tenía diagnóstico definido en el informe anatomo-patológico original. Se refiere a una niña de ocho años que fue operada en julio de 1938 por un tumor abdominal, en el Hospital de La Misericordia, y del cual se toma biopsia al no poderse extraer.

En los quistes dermoides generalmente se encuentran tejidos derivados de la hojilla ectodérmica, como pelos, dientes, etc., pero también hay estructuras variables meso o endodérmicas; tienen una frecuencia de 10 al $15 \%$ de todos los tumores ováricos. Los teratomas inmaduros son muy raros y en cuanto a su malignidad, parece que en los jóvenes el crecimiento rápido del tumor es mejor índice para juzgar dicha malignidad, en cada caso individual (Lisa, op. cit.). Potencialmente los dermoides pueden malignizarse; se señala de un 3 a un $8 \%$ de estos casos. La degeneración sarcomatosa es la más común, conforme a la evidencia reconocida en jóvenes; la degeneración carcinomatosa es más frecuente en adultos (Lisa, op. cit.).

Cuando el teratoma esboza la organización de un embrión reconocible, aunque incompletamente formado, se le llama embrioma. En la literatura se han citado algunos casos, como el estudiado por Brunkow, Hunter y otros (citados por Schauffler) (67), en una niña de catorce meses y el cual al ser publicado en la prensa sin autorización, se sugería la posibilidad de un embarazo precoz. Al operar se encontró un monstruoso feto, con vértebras, costillas, huesos comparables a las extremidades superiores e inferiores, rodeados de músculos esqueléticos y todo el conjunto del tumor recubierto por piel. Había sistema nervioso, ganglios simpáticos, intestino bien desarrollado, tracto urinario, suprarrenales y lo más interesante, un ovario en desarrollo. Había pues, una tentativa claramente ordenada de reproducción de otro cuerpo.

A la Academia de Medicina de París se presentó este año un caso similar, ocurrido en Francia y al cual le dio también amplia publicidad la prensa. 


\section{CUADRO NUMERO 3}

OVARIOS

\begin{tabular}{|c|c|c|c|}
\hline Quiste simple & 5 & $\ldots \ldots$ & $17.24 \%$ \\
\hline 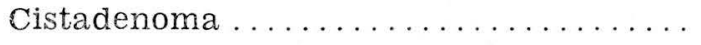 & 2 & $\cdot$ & $6.89 \%$ \\
\hline Carcinoma primitivo ............. & 6 & $\ldots \ldots$ & $20.68 \%$ \\
\hline Tumor de Krukemberg . ............ & 1 & $\ldots$ & $3.44 \%$ \\
\hline Sarcoma. . . . . . . . . . . . . . & 1 & $\ldots$ & $3.44 \%$ \\
\hline 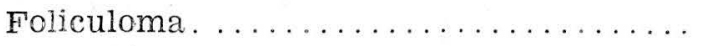 & 1 & $\ldots \ldots$ & $3.44 \%$ \\
\hline 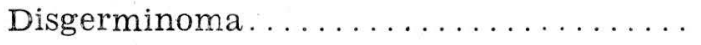 & 2 & $\ldots \ldots$ & $6.89 \%$ \\
\hline Quiste dermoide ..... & 10 & $\ldots \ldots$ & $34.48 \%$ \\
\hline Teratoma maligno .... & 1 & $\ldots \ldots$ & $3.44 \%$ \\
\hline TOTAL. & 29 & & $100 \%$ \\
\hline
\end{tabular}

\section{CUADRO NUMERO 4.}

RELACION GENERAL ENTRE TUMORES MALIGNOS

Y BENIGNOS

\begin{tabular}{|c|c|c|}
\hline Benignos: & Malignos: & \\
\hline Anginomas g. ext. ....... 5 & Sarcomas vulva ........ & 3 \\
\hline Papilomas g. ext. . . . . . . . 1 & Sarcomas ov. ........... & 1 \\
\hline Quistes simples ov. ........ 5 & Carcinomas ut. ......... & 1 \\
\hline Cistadenomas ov. ..... & Carc prim ov. ......... & 6 \\
\hline \multirow[t]{4}{*}{ Quistes dermoides ov. } & Carc. sec. ov. ......... & 1 \\
\hline & Foliculomas .......... & 1 \\
\hline & Disgerminomas........... & 2 \\
\hline & Teratomas malig. ov. ..... & 1 \\
\hline TOTALES. & & 16 \\
\hline
\end{tabular}

BENIGNOS: $58.98 \%$. MALIGNOS: $41.02 \%$.

\section{SUMARIO}

Por primera vez en Colombia se hace un estudio sobre las ginecopatías tumorales en la niñez, se revisa la literatura sobre el particular y se presenta una serie de 39 casos.

Se hace un resumen breve de las nociones elementales de Oncología, clasificaciones, etc., algunas consideraciones generales 
sobre los tumores en la niñez y en especial sobre las neoplasias del aparato genital de la niña.

Se analizan los tumores de los diversos segmentos del aparato genital, se describen los tumores más frecuentes, se discuten los casos encontrados y se dan frecuencias sobre ellos.

Especial importancia se le asigna a un discutido caso de sarcoma botryoides de la vulva y se revisa la literatura al respecto.

En este trabajo de tesis y por primera vez entre nosotros, se publican dos casos de disgerminoma del ovario en niñas.

Se llama la atención para no olvidar estas lesiones en el diagnóstico diferencial, se hace énfasis sobre ello, y se insiste en que no se deben considerar infrecuentes las ginecopatías tumorales en la niñez.

\section{CONCLUSIONES}

Los tumores genitales de la niñez tienen, en relación con todas las ginecopatías tumorales, una frecuencia de $0.703 \%$, es decir, cerca del $1 \%$.

Las neoplasias ginecológicas más frecuentes en las niñas son los tumores del ovario, con una frecuencia del $74.35 \%$ de los casos estudiados.

En segundo orden están las neoplasias de los genitales externos, con una frecuencia del $23.07 \%$.

No se encontraron neoplasias de la vagina y para el útero se deduce una frecuencia del $2.36 \%$.

En el ovario de las neoplasias más comunes son:

El quiste dermoide $(34.48 \%)$, el carcinoma primitivo $(20.68 \%)$ y el quiste de retención $(17.24 \%)$.

En los genitales externos la neoplasia más común es el angioma $(55.55 \%)$, le sigue el sarcoma de la vulva $(33.33 \%)$.

El quiste dermoide del ovario es el tumor genital más frecuente en niñas $(25.64 \%$ de todos los tumores genitales y $34.48 \%$ de los tumores del ovario).

El cáncer primitivo del ovario es la neoplasia maligna más frecuente en el aparato genital de las niñas y en segundo lugar, el sarcoma de la vulva.

Señalamos la alta frecuencia del cáncer genital de las niñas $(41.02 \%)$ en nuestro medio, que casi iguala la frecuencia de las rieoplasias benignas $(58.98 \%)$ del aparato genital. 
Llama la atención que las neoplasias en las niñas tiendan a localizarse en los dos extremos del tracto genital: los genitales externos y los ovarios.

Llama la atención que el sarcoma tienda a localizarse en la vulva de las niñas y el carcinoma en la vulva de las mujeres adultas de edad avanzada.

Llama la atención que el sarcoma de la vulva tienda a ocurrir en los primeros meses de la vida.

Llama la atención que el carcinoma primitivo del ovario tienda a ocurrir en la primera mitad de la segunda década de la vida.

La torsión es la complicación más frecuente de los tumores del ovario; pueden ocurrir a cualquier edad y en todo tipo de tumor.

Hacemos notar la ausencia de bibliografía nacional.

Comunicamos los dos primeros casos de disgerminoma del ovario en niñas, con lo cual sube a cinco el número de casos en total publicados en el país.

No tenemos noticias si de los otros tipos de neoplasias se haya publicado entre nosotros algún caso en niñas. 


\section{BIBLIOGRAFIA}

1. BONILLA-NAAR A.- Bases para escribir un trabajo científico, Rev. Col. Obst. y Ginec., 4: 155, junio, julio, 1953.

2. EWING JAMES.- Oncología, IV. Ed.; 10, 1948.

3. VON RIND-FLEISCH (Cit. de Ewing: 10).

4. ZIEGLER.- Lehorb. Path. Anat., 1898 (Cit. de Ewing).

5. BIRSCH-HIRSCHFELD.- Path. Anat., 1896 (Cit. de Ewing).

6. RIBBERT.- Das path. Wachtuur, Geschwnestbildung, Bonn, 1896 (cit. de Ewing).

7. LUBARSCH.-- Erg. Path. Anat.: 1895 (Cit. de Ewing).

8. BORST.-Die Lehre v. dd. Gesch, 1902 (Cit. de Ewing).

9. WHITE (citado por Ewing: 10).

10. DARGEON H. W.- Cancer in Childhood: 58, 1940.

11. PACK G.- Sarcomas of the soft somatic tissues in infants and children, Surg. Gynec. \& Obst., 98: 675, junio, 1954.

12. DARGEON H. W.- Cancer in children from birth to fourteen years of age, J. A. M. A., 136: 459, febrero 1948.

13. GORDON ROBERTSON G.- Embrylogic development of the female genital system, pediatric Gynecology, Schauffler, third edition: 24, 1953.

14. APARICIO J. A.- Clínica Ginecológica, conferencias: 96, 1953.

15. GURLT (citado por Dargeon).

16. ARGUELLES R.- Manual de Patología Quirúrgica: 211, 1950.

17. RIWERT.- Cancer in childhood (cita de Dargeon).

18. THOMA.- Cancer in childood (cita de Dargeon: 459).

19. GOMEZ FORERO J.- Observaciones de tumores en niños de edad pre-escolar, tesis de grado, 1952.

20. COSTERO A.- Tratado de Anat. Pat.: 828, 1950.

21. WAGNER.- Am. J. Obst. \& Gynec (cita de Spademan) 65: 203, enero 1953.

22. SPIEGELEERG. (citado por Spademan).

23. SPADEMMAN L. C. et al.- Sarcoma botryoides, Am. J. Obst. \& Gynec. 65: 203, enero 1953.

24. SIMPSON. J. Obs. \& Gynec (cita de Spademan).

25. CONILL-MONTOBIO V.-- Tratado de Ginecología y de Técnica Terapéutica Ginecológica: 435, 1946.

26. Mc. FARLAND, J.- Dysontogenetic and Mixed tumors of the urogenital region, with report of new case of sarcoma botryoides vaginae in child, and comments upon probable nature of sarcoma. Surg. Gynec \& Obst., 61: $42-57,1935$.

27. GLASS, M. et all.- Am. J. Obst. and Gynec. 41: 309, 1941.

28. RICHMOND E. L. - Sarcnma botryoides of cervix, A. J. Obst. \& Cynec., 65: 201, enero de 1953.

29. ULFELDER (citado por Richmond).

30. STOECKEL W.- Lehrbuch Der Gynakologie; 167, 1952.

31. SADLER W. P. and DOCKERTY.- Malignant Mioblastoma vulvae, Am. J. Obst. \& Gynec. 61: 1047-1055, 1951.

32. KELLER J.- Fibrosarcoma of labium vulvae, Canad. M. A. J. 64: 534$536,1951$.

33. OBER W. B. et all.- Rhabdomiosarcoma of the vulva and vagina, A. M. A. arch. of pathology, 56:3640-373, 1953.

54. WILLIAMS (citado por Ewing).

35. WINCKEL (citado por Ewing). 
36. BALDWIN L. G.- Am. J. Obst. \& Ginec., 31: 728, 1931.

37. SCHAUFF'LER GOODRICH C.- Pediatric Gynecology, Third edition, Year Book Publishers, Chicago; 128, 1953.

38. CRAWFORD (citado por Dargeon).

39. BONNER A.- Am. J. Obst. \& Gynec., 14: 175, 1927.

40. GILBERT J. B.- Am. J. Obst. \& Gynec., 24: 402, 1935.

41. LOCKHART H.- Am. J. Obst. \& Gynec., 30: 76, 1935.

42. JAMES T.- A. benign polipoid tumour of the cervix uteri in a girl three years old, J. of Obst. \& Ginec. of Brithis Empire, 58: 160, octber, 1951.

43. HARNED J. et all.- Childhood Cancer, J. of Pediatric., 42: 612, mayo, 1953.

44. DORAND (citado por Ewing).

45. LONNEBERG (citado por Ewing).

46. WEILL H. I.- Bull. John Hpkins, 16: 102, 1905 (citado por Ewing).

47. HUBERT (citado por Ewing).

48. HALL N. and BAGBY J. W.- J. A. M. A., 110: 703, 1938.

49. LESH (citado por Schauffler).

50. HUNT V. C.- Am. Surg., 87: 84, 1928.

51. DOWNES W. A.- J. A. M. A., 76: 443, 1921.

52. KENNL (citado por Ewing).

53. LEVI A. A.- New England, J. Med., 217: 595, 1935.

54. LISA J. R.- Teratomas ováricos malignos en las dos primeras décadas de la vida, Am. J. Surg., 81: 453, abril, 1951.

55. DARGON H. W.- Cancer in childhood and a discussion of certain benign tumors, 1940.

56. POWNES (citado por Lisa).

57. PEDOWITZ P. and GRAYZEL D. M.- Disgerminoma del ovario, Am. J. Obst. \& Gynec, junio, 1951.

58. LOPEZ ESCOEAR G.- Tumores del ovario, 1952.

59. GOLDSTEIN S.- Embryonic carcinoma of the ovary in a girl 13 years of age, Am. J. Odst. \& Gynec, 40: 867, 1940.

60. SAGER W. W.- Am. J. Obst. \& Gynec., 40: 867, 1940.

61. GOODALL J. R.- Tumors of the ovary. Del libro de Curtis. Obstetrics and gynecology. 1937.

62. DIAZ SARDIÑA y otros.- Sobre un caso de sarcoma del ovario, arch. cubanos de cancerología, 4: 934, 1945.

63. NOVAK E.- Am. J. Obst. y Gynec., 26: 505, 1933.

64. LOPEZ ESCOBAR G.- Arrenoblastoma ovárico, Med. y Cir., 14: 199, abril, 1950.

65. PINTO J.-Algunas consideraciones sobre el ovario y sus tumores, tesis Fac. Med. 1932 (citado por López E.).

66. AMOROCHO, LICHTENBERGER y BECERRA.- Disgerminoma del ovario, rev. Fac. Med. Bog., 17: 610, junio, 1949.

67. BRUNKOW (citado por Schauffler). 